\title{
The compression theorem II: directed embeddings
}

\author{
Colin Rourke \\ BRIAN SANDERSON \\ Mathematics Institute, University of Warwick \\ Coventry, CV4 7AL, UK \\ Email: cpr@maths.warwick.ac.uk and bjs@maths.warwick.ac.uk \\ URL: http://www.maths.warwick.ac.uk/ cpr/ and bjs/
}

\begin{abstract}
This is the second of three papers about the Compression Theorem. We give proofs of Gromov's theorem on directed embeddings $\left[1 ; 2.4 .5\left(\mathrm{C}^{\prime}\right)\right]$ and of the Normal Deformation Theorem [3;4.7] (a general version of the Compression Theorem).
\end{abstract}

AMS Classification numbers Primary: 57R40, 57R42

Secondary: 57A05

Keywords: Compression, flattening, directed embedding, ripple lemma

Proposed: Robion Kirby

Seconded: Yasha Eliashberg, David Gabai

(C) Geometry $8 \mathcal{T}$ Topology $\mathcal{P}$ ublications
Received: 25 January 2001

Revised: 2 April 2001 


\section{Introduction}

This is the second of three papers about the Compression Theorem. The first paper [3] contains a proof of the theorem using an explicit vector field argument. This paper contains one simple piece of geometry, rippling, which proves the "flattening lemma" stated below. This leads to proofs of Gromov's theorem on directed embeddings $\left[1 ; 2.4 .5\left(\mathrm{C}^{\prime}\right)\right]$ and of the Normal Deformation Theorem $[3 ; 4.7]$ (a general version of the Compression Theorem which can be readily deduced from Gromov's theorem). The third paper [4] is concerned with applications.

We work throughout in the smooth $\left(C^{\infty}\right)$ category and we shall assume without comment that all manifolds are equipped with appropriate Riemannian metrics. The tangent bundle of a manifold $W$ is denoted $T W$. Suppose that $M^{n}$ is smoothly embedded in $Q^{q} \times \mathbb{R}^{t}$ where $q \geq n$. We think of $\mathbb{R}^{t}$ as vertical and $Q$ as horizontal. We say that $M$ is compressible if it is nowhere tangent to vertical, or equivalently, if projection $p$ on $Q$ takes $M$ to an immersion in $Q$. Throughout the paper, "normal" means independent (as in the usual meaning of "normal bundle") and not necessarily perpendicular.

Let $G=G_{n}\left(Q^{q} \times \mathbb{R}^{t}\right)$ denote the Grassmann bundle of $n$-planes in $T\left(Q^{q} \times \mathbb{R}^{t}\right)$ and define the horizontal subset $H$ of $G$ to comprise $n$-planes with no vertical component. In other words $H$ comprises $n$-planes lying in fibres of $p^{*} T Q$.

Flattening lemma Suppose that $M^{n}$ is compressible in $Q^{q} \times \mathbb{R}^{t}$ and that $U$ is any neighbourhood of $H$ in $G$. Suppose that $q-n \geq 1$ then there is a $C^{0}$-small isotopy of $M$ in $Q$ carrying $M$ to a position where $T M \subset U$.

We think of planes in $H$ as flat and planes in $U$ as almost flat. So the lemma moves $M$ to a position where it is almost flat (ie, its tangent bundle comprises almost flat planes). Obviously it is in general impossible to move $M$ to a position where it is completely flat.

\section{Addenda}

(1) The lemma is also true if $q=n$ and each component of $M$ is either open or has boundary. However in this case the isotopy is not $C^{0}$-small, but of the form "shrink to a neighbourhood of a (chosen) spine of $M$ followed by a small isotopy". We call such an isotopy "pseudo-small"; a pseudo-small isotopy has support in a small neighbourhood of the original embedding.

(2) The lemma has both relative and parametrised versions: If $M$ is already almost flat in the neighbourhood of some closed subset $C$ of $M$ then the isotopy 
can be assumed fixed on $C$. Further given a family of compressible embeddings of $M$ in $Q$ parametrised by a manifold $K$, then there is a $K$-parametrised family of small isotopies of $M$ in $Q$ carrying $T M \times K$ into a given neighbourhood of $H \times K$.

(3) In the non-compact case smallness can be assumed to vary. In other words, during the isotopy, points move no further than $\varepsilon>0$, which is a given function of $M$ (or $M \times K$ in the parametrised version).

\section{The proof of the flattening lemma}

The process is analogous to the way in which a road is constructed to go up a steep hill. Hairpin bends are added (which has the effect of greatly increasing the horizontal distance the road travels) and this allows the slope of the road to become as small as necessary.

The process of adding hairpin bends is embodied in the "ripple lemma" which we state and prove first. The flattening lemma follows quickly from the ripple lemma.

Let $M$ be connected and smoothly immersed in $Q$. Suppose that $Q$ has a Riemannian metric $d$. Define the induced Riemannian metric denoted $d_{M}(x, y)$ on $M$ by restricting to $T M$ the form on $T Q$ which defines $d$.

If $M$ is embedded in $Q$ then we also have the usual induced metric on $M$ (ie $d$ restricted to $M$ ). The two induced metrics coincide to first order for nearby points but in general the induced metric is smaller than the induced Riemannian metric.

Ripple lemma Suppose that $M$ is smoothly embedded in $Q$ and that $q-n \geq$ 1 and that $R, \varepsilon>0$ are any given real numbers. Then there is an isotopy of $M$ in $Q$ which moves points at most $\varepsilon$ such that the finishing diffeomorphism $f: M \rightarrow f(M)$ has the following property:

$$
d_{f(M)}(f(x), f(y))>R d_{M}(x, y) \text { for all } x, y \in M
$$

Thus the ripple lemma asserts that we can (by a small isotopy) arrange for distances of points (measured using the induced Riemannian metric from $Q$ ) to be scaled up by as large a factor as we please. The proof is to systematically "ripple" the embedding, hence the name. 
Proof We deal first with the case when $Q$ is the plane and $M$ is the unit interval $[0,1]$ in the $x$-axis, so $n=1$ and $q=2$. We will work relative to the boundary of $M$; in other words the isotopy we construct will be fixed near 0 and 1 and the scaling up will work for points outside a given neighbourhood of $\{0,1\}$.

Consider a sine curve $S$ of amplitude $A$ and frequency $\omega$ (the graph of $y=$ $A \sin (2 \pi \omega x))$. Think of $\omega$ as large and $A$ as small. So the curve is a small ripple of high frequency. Use a $C^{\infty}$ bump function to phase $S$ down to zero near $x=0$ and $x=1$. See figure 1 .

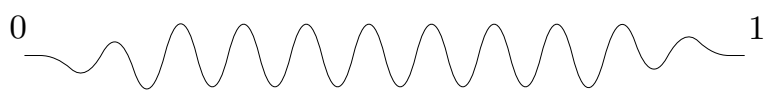

Figure 1: The basic ripple

Clearly $M$ can be replaced by $S$ via an isotopy which moves points at most $A+\frac{1}{\omega}$. Further we can choose this isotopy to finish with a diffeomorphism which nowhere shrinks distances, is fixed near $\{0,1\}$, and which outside a given neighbourhood of $\{0,1\}$ scales length up (measured in $M$ ) by a constant scale factor.

But the length of $S$ is greater than $4 A \omega$ since the distance along the curve through one ripple is greater than $4 A$. Thus by choosing $A$ sufficiently small and then choosing $\omega$ sufficiently large the lemma is proved (relative to the boundary) in this special case.

For future reference, we shall denote the 1-dimensional rippling diffeomorphism, just constructed, by $r$. Or to be really precise, we use $r$ to denote this rippling diffeomorphism without phasing out near $\{0,1\}$.

For the general case, we use induction on a handle decomposition of $M$. Here is a slightly inaccurate sketch of the procedure. At each step of the induction we move one handle keeping fixed the attaching tube. The handle gets replaced by a neighbourhood of its core (and there is a compensatory enlargement of handles attached onto it) otherwise the decomposition remains fixed throughout the induction. For each handle in turn we think of the core as a cube, choose one direction in the cube and one direction perpendicular to the core. Then, using the model in $\mathbb{R}^{2}$ just given, we ripple the chosen direction in the core, crossing with the identity on other coordinates and phasing out near the boundary of the core. Then we choose another direction in the cube and repeat this move. This has the effect of creating perpendicular, and smaller, ripples on top of the ripples just made (figure 2). We repeat this for each direction in the cube 
and the end result is that all distances (in the core) are scaled. We then scale distances in the handle near the core by expanding a very small neighbourhood of the core onto a small one. Finally we redefine the handle to lie inside the very small neighbourhood (changing the handle decomposition of $M$ ) and proceed to the next handle.

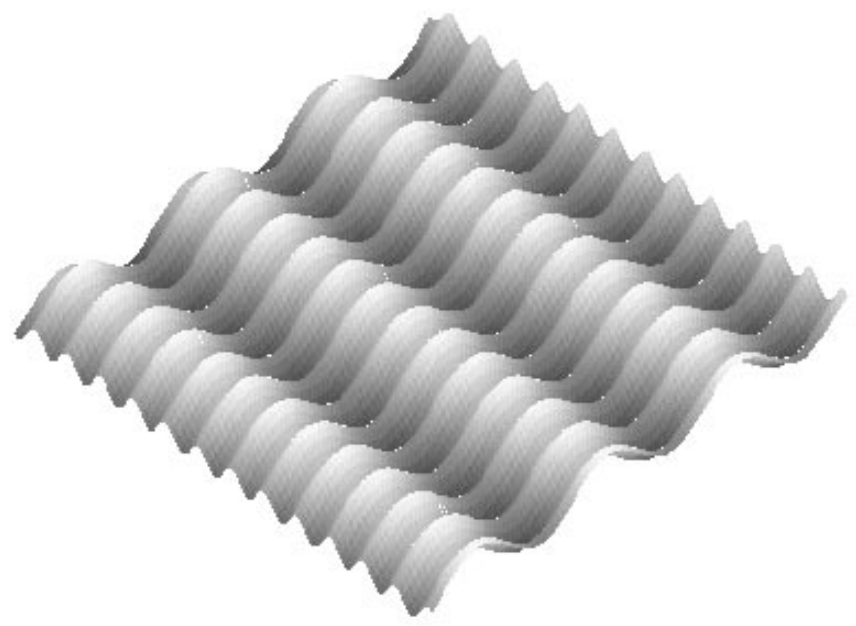

Figure 2: The effect of two successive ripples in the middle of the core

\section{The model}

For the details, let $I^{j}$ be the standard $j$-cube in $\mathbb{R}^{j}$. We shall construct a model ripple $r_{j}$ of $I^{j}$ in $I^{j} \times \mathbb{R}$ by using the standard 1-dimensional ripple $r$, defined earlier, $j$ times. Let $T$ be a given constant and let $I_{0}^{j} \subset \operatorname{int} I^{j}$ be a given concentric copy of $I^{j}$. Let $\phi$ be a bump function which is 1 on $I_{0}^{j}$ and 0 outside a compact subset of $\operatorname{int} I^{j}$.

For each $t=1,2, \ldots, j$ we perform the following inductive move. Starting with $t=1$ we ripple $I^{j}$ by using $r$ on the first coordinate and the identity on the remaining $j-1$ and phase out by using $\phi$. We choose the parameters for $r$ to scale distances by the the given constant $T$. This defines a subset $I_{1}^{j}$ of $I^{j} \times \mathbb{R}$ and a diffeomorphism $r_{1}: I^{j} \rightarrow I_{1}^{j}$ which stretches distances in $I_{0}^{j}$ in the direction of the first coordinate by the factor $T$. Now suppose inductively that $r_{t-1}: I^{j} \rightarrow I_{t-1}^{j}$ has been defined. Choose a small orthogonal normal bundle on $I_{t-1}^{j}$ in $I^{j} \times \mathbb{R}$ and use this and $r_{t-1}$ to identify a neighbourhood $U$ of $I_{t-1}^{j}$ with $I^{j} \times V$ where $V$ is an open interval in $\mathbb{R}$ containing 0 . Using this identification define $s_{t}: I_{t-1}^{j} \rightarrow U$ by using exactly the same construction as used for $r_{1}$, but replacing the first coordinate by the $t^{\text {th }}$ and choosing the height of the sine 
function sufficently small that the image lies in $U$, and adjusting the frequency so that the scale factor is again $T$. Define $I_{t}^{j}=s_{t}\left(I_{t-1}^{j}\right)$ and $r_{t}=s_{t} \circ r_{t-1}$.

Now throughout this inductive process, the coordinate system for $r_{t}\left(I^{j}\right)$, coming from the standard coordinate system for $I^{j}$, remains perpendicular on $I_{0}^{j}$. It follows that $r_{j}$ scales all distances in $I_{0}^{j}$ by factor approximately $T$. The reason why the factor is not exactly $T$ is because, after the first ripple, $I_{1}^{j}$ is not flat. Hence there is a shrinkage effect because moving out in the $\mathbb{R}$-direction can move points closer together. By choosing $U$ sufficiently small, this effect can be made as small as we please. Hence all distances in $I_{0}^{j}$ are stretched by a factor, which may vary with direction, but which is uniformly as close as we like to $T$.

Another effect occurs outside $I_{0}^{j}$. Here the non-constant scaling in say the first coordinate (due to the phasing to zero using $\phi$ ) causes the second coordinate system to become non-perpendicular and hence the second scaling may in fact shrink some distances. But it can be checked that this shrinking is by a factor $\geq \sin (\alpha)$ where $\alpha$ is the minimum angle between the images of two lines at right angles. But by choosing the size of all the ripples to be small compared to the distance between $\partial I_{0}^{j}$ and $\partial I^{j}$ (in other words the distance over which $\phi$ varies) the distortion in the coordinate system can be made as small as we please and hence $\sin (\alpha)$ chosen as near as we like to 1 . The way to think of this is that we are using small ripples whose height varies over a much larger scale. Thus by choosing the parameters carefully we can assume that, in the model ripple $r_{j}$, all distances in $I_{0}^{j}$ are scaled up by factor as close to $T$ and as nearly independent of direction as we please. Outside $I_{0}^{j}$ distances are scaled up by a factor which varies from point to point, but which, at a given point is again as nearly independent of direction as we please.

This completes the construction of the model.

Now to prove the lemma choose a finite (or locally finite) handle decomposition of $M$ and suppose that $M_{1}=M_{0} \cup h_{j}$ (where $h_{j}$ is a handle of index $j$ ). Suppose inductively that for given $\delta>0$ we have constructed an isotopy finishing with a diffeomorphism $f_{0}$ which moves points at most $\delta$ such that property $(*)$ holds near $M_{0}$ with $f_{0}$ in place of $f$.

Now choose a diffeomorphism $g$ of $\operatorname{int} I^{j}$ with the core of $h_{j}$ minus attaching tube and let $I_{0}^{j}$ be chosen so $g\left(\operatorname{int} I^{j}-I_{0}^{j}\right)$ is contained in the neighbourhood of $M_{0}$ where $(*)$ already holds. Choose a small orthogonal line bundle $\zeta$ on $h_{j}$ minus attaching tube in $Q$ and use this to extend $g$ to an embedding of int $I^{j} \times V$ where $V$ is an open interval in $\mathbb{R}$ containing 0 . Define $f_{1}$ to be $g \circ r_{j} \circ g^{-1}$, where $r_{j}$ is the model ripple contructed above. Then, by choosing $T$ sufficiently large (noting that the directional derivatives of $g$ are bounded on a compact 
subset of $\left.\operatorname{int} I^{j}\right), f_{1}$ satisfies property $(*)$ for points in a neighbourhood of $M_{0}$ and in the core. There is again a shrinkage effect due to the fact that $h_{j}$ is not flat (hence moving out along $\zeta$ can move points closer together). By choosing $\zeta$ sufficiently small, this effect can again be made as small as we please. To stretch distances perpendicular to the core in the handle we choose a small neighbourhood $V$ of the core containing a much smaller neighbourhood $V^{\prime}$. Then the isotopy which expands $V^{\prime}$ onto $V$ stretches distances perpendicular to the core. Finally we change the handle decomposition of $M$ by redefining $h_{j}$ to lie inside $V^{\prime}$ (this could be done by a diffeomorphism of $M$ and hence defines a new decomposition). We now have property $(*)$ in a neighbourhood of $M_{1}$. Note that the finite number of small moves used on $h_{j}$ can be assumed to move points at most any given $\delta^{\prime}$. Thus by choosing successive moves bounded by a sequence which sums to less than $\varepsilon$, lemma is proved by induction.

Addenda The proof of the ripple lemma leads at once to various extensions:

(1) There is a codimension 0 version (ie $q=n$ ) as follows. Let $X$ be a spine of $M$. Choose a handle decomposition with cores lying in $X$ and no $n$-handles. Apply the proof to this decomposition. We obtain a neighbourhood $N$ of $X$ in $M$ and a small isotopy of $N$ in $Q$ such that $(*)$ holds in $N$.

(2) In the non-compact case we can assume that $R$ and $\varepsilon$ are arbitrary positive functions. (This follows at once from the local nature of the proof.)

(3) The proof gives both relative and parametrised versions. In the relative version we can assume that the isotopy is fixed on some closed subset and obtain $(*)$ outside a given neighbourhood of $C$. (This follows at once from the method of proof.) In the parametrised version we are given a family of embeddings parametrised by a manifold $K$ and obtain a $K$-parameter family of small isotopies such that $(*)$ holds for each finishing embedding, where both $R$ and $\varepsilon$ are functions of $K$. To see this, we use the same scheme of proof but at the crucial stage we choose a diffeomorphism of a neighbourhood of (the core of $\left.h_{j}\right) \times K$ in $Q \times K$ with a neighbourhood of $I^{j} \times K$ in $\mathbb{R}^{q} \times K$. We then use the same model rippling move over each point of $K$ varying the controlling parameters appropriately. The rest of the proof goes through as before.

(4) Since the process is local, there is an immersed version of the lemma in which $M$ is immersed in $Q$ and a regular homotopy is obtained. Further all these extensions can be combined in obvious ways.

Proof of the flattening lemma We can now deduce the flattening lemma. What we do is ripple the horizontal coordinate using the immersed version of the ripple lemma. This has the effect that horizontal distances are all scaled 
up. We leave the vertical coordinate fixed. The embedding is now as flat as we please. The addenda to the flattening lemma follow from the addenda to the ripple lemma given above.

In order to prove the Normal Deformation Theorem (in the next section) we shall need a bundle version of the lemma:

Bundle version of the flattening lemma Suppose that $M^{n} \subset W^{t+q}$ where $q-n \geq 1$ and $T W$ contains a subbundle $\xi^{t}$ (thought of as vertical) such that $\xi \mid M$ is normal to $M$. Let $H$ (the horizontal subset) be the subset of $G_{n}(W)$ of $n$-planes orthogonal to $\xi$. Then given a neighbourhood $U$ of $H$ in $G_{n}(W)$ there is a $C^{0}$-small isotopy of $M$ in $Q$ carrying $M$ to a position where $T M \subset U$.

Proof Use a patch by patch argument. Approximate locally as a product $\mathbb{R}^{t} \times$ patch and use the (relative) $\mathbb{R}^{t}$ version.

Remarks (1) There are obvious extensions to the bundle version corresponding to the extensions to the $\mathbb{R}^{t}$ version. For the proofs we use a similar patch by patch argument together with the appropriate extension of the $\mathbb{R}^{t}$ version.

(2) Rippling has been used on occasions by several previous authors, in particular Kuiper used it (together with some sophisticated estimates) to prove his version of the Nash isometric embedding theorem [2].

\section{Normal deformations and Gromov's theorem}

Gromov's theorem asserts (roughly) that if $M \subset W$ and the tangential information is deformed within a neighbourhood of $M$ (ie, $T M$ is deformed as a subbundle of $T W$ ) then the deformation can be followed within a neighbourhood by an isotopy of $M$ in $W$. We shall prove the theorem in the following equivalent normal version (ie, $M$ follows a deformation of a bundle normal to $M$ in $W$ ). The normal version follows quickly from a repeated application of the flattening lemma. We shall give a precise statement (and deduction) of Gromov's theorem after this version.

Normal Deformation Theorem Suppose that $M^{n} \subset W^{w}$ and that $\xi^{t}$ is a subbundle of $T W$ defined in a neighbourhood $U$ of $M$ such that $\xi \mid M$ is normal to $M$ in $W$ and that $t+n<w$. Suppose given $\varepsilon>0$ and a homotopy of $\xi$ through subbundles of $T W$ defined on $U$ finishing with the subbundle $\xi^{\prime}$. Then there is an isotopy of $M$ in $W$ which moves points at most $\varepsilon$ moving $M$ to $M^{\prime}$ so that $\xi^{\prime} \mid M^{\prime}$ is normal to $M^{\prime}$ in $W$. 
Proof Suppose first that $M$ and $U$ are compact. It follows that the total angle that the homotopy of $\xi$ moves planes is bounded and we can choose $r$ and a sequence of homotopies $\xi=\xi_{0} \simeq \xi_{1} \simeq \ldots \simeq \xi_{r}=\xi^{\prime}$ so that for each $s=1, \ldots, r-1$ the planes of $\xi_{s-1}$ make an angle less than $\pi / 4$ with those of $\xi_{s}$. We apply (the bundle version of) the flattening lemma $r+1$ times each time moving points of $M$ at most $\frac{\varepsilon}{r+1}$. Start by flattening $M$ to be almost perpendicular to $\xi=\xi_{0}$. Then $\xi_{1}$ is now normal to $M$ and we flatten $M$ to be almost perpendicular to $\xi_{1}$. $\xi_{2}$ is now normal to $M$ and we flatten $M$ to be almost perpendicular to $\xi_{2}$ etc. After $r+1$ such moves $M$ has been moved to $M^{\prime}$ say which is almost perpendicular to $\xi_{r}=\xi^{\prime}$ and in particular is normal to it.

For the non-compact case, argue by induction over a countable union of compact pieces covering $M$.

\section{Addenda}

The proof moves $M$ to be almost perpendicular to $\xi^{\prime}$ (not just normal). Further it can readily be modified to construct an isotopy which "follows" the given homotopy of $\xi$, in other words if $\xi_{i}$ is the position of $\xi$ at time $i$ in the homotopy and $M_{i}$ the position of $M$ at time $i$ in the isotopy, then $\xi_{i} \mid M_{i}$ is normal to $M_{i}$ for each $i$. To see this reparametrise the time for the isotopy so that the flattening of $M$ with respect to $\xi_{i}$ takes place near time $\frac{i}{r}$. This produces a rather jerky isotopy following the given bundle homotopy but by breaking the homotopy into very small steps, the isotopy becomes uniform and furthermore (apart from the initial move to become almost perpendicular to $\xi$ ) $M_{i}$ is almost perpendicular to $\xi_{i}$ throughout.

There are obvious relative and parametrised versions similar to those for the flattening lemma (and proved using those versions), and furthermore we can assume that $\varepsilon$ is given by an arbitrary positive function in non-compact cases. Finally there is a codimension $0(t+n=w)$ version which it is worth spelling out in detail, since this is the version that implies Gromov's theorem:

Suppose in the Normal Deformation Theorem that $t+n=w$ and that $M$ is open or has boundary and that $X$ is a spine of $M$. Then there is an isotopy of $M$ of the form: shrink into a neighbourhood of $X$ followed by a small isotopy in $W$, carrying $M$ to be almost perpendicular to $\xi^{\prime}$.

Gromov's Theorem $\left[1 ; 2.4 .5\left(\mathrm{C}^{\prime}\right)\right.$, page 194] Suppose that $M^{n} \subset W^{w}$ and suppose that $M$ is either open or has boundary and that we are given a deformation of $T M$ over the inclusion of $M$ to a subbundle $\eta$ of $T W$. Then there is an isotopy of $M$ carrying TM into a given neighbourhood of $\eta$ in the Grassmannian $G_{n}(W)$. 
Proof Let $\xi$ be the orthogonal complement of $T M$ in $T W$. Then the deformation of $T M$ gives a deformation of $\xi$ to $\xi^{\prime}$ say. Pulling the bundles back over a neighbourhood of $M$ gives the hypotheses of the Normal Deformation Theorem (codim 0 case above). The conclusion is the required isotopy.

Final remarks It is easy to reverse the last argument and deduce the Normal Deformation Theorem from Gromov's Theorem.

Another proof of the Normal Deformation Theorem is given in [3] as an extension of the arguments used to prove the Compression Theorem. The proofs in [3] are quite different in character from those presented here. We think of the bundles locally as defined by independent vector fields and define flows by extending these vector fields in an explicit fashion. The resulting embeddings are far more precisely defined: indeed instead of a multiplicity of ripples, there is in the simplest case a single twist created around a certain submanifold which we call the downset. For details here see the pictures in section 3 and the arguments in section 4 of [3].

We are very grateful to both Yasha Eliashberg and the referee for comments which have greatly improved the clarity of the proof of the ripple lemma.

\section{References}

[1] M Gromov, Partial differential relations, Springer-Verlag (1986)

[2] Nicolaas H Kuiper, On $C^{1}$-isometric imbeddings. I, II, Nederl. Akad. Wetensch. Proc. Ser. A. 58 = Indag. Math. 17 (1955), 545-556 and 683-689

[3] Colin Rourke, Brian Sanderson, The compression theorem I, Geometry and Topology, 5 (2001) 399-429, arxiv:math.GT/9712235

[4] Colin Rourke, Brian Sanderson, The compression theorem III: applications, to appear, see http://www.maths. warwick.ac.uk/ ${ }^{\sim} \mathrm{cpr} / \mathrm{ftp} / \mathrm{compIII.ps}$ for a preliminary version 